\title{
ABORDAGEM DISCRETA PARA A DINÂMICA DA TAXA SELIC-META
}

\author{
Sidney Martins Caetano* \\ Geraldo Edmundo Silva Jr. ${ }^{\dagger}$ \\ Wilson Luiz Rotatori CORRÊA
}

\begin{abstract}
Resumo
O presente artigo utiliza abordagem discreta não estacionária para modelar taxa de juros no Brasil. O diferencial está em abordar o problema em termos das decisões de alta, baixa ou manutenção da taxa Selic tomadas pelo Copom como uma decisão discreta ao invés de contínua. O modelo proposto se mostra parcimonioso e capaz de prever $83 \%$ das decisões do Copom. O modelo permite que os parâmetros estimados que definem o limiar para o qual o Copom tomaria a decisão sejam assimétricos, mas não encontra diferenças significativas entre os limiares de alta e baixa.
\end{abstract}

Palavras-chave: Política Monetária; Regra de Taylor; Abordagem Discreta Não Estacionária.

JEL classification: C25, E52, E58

\begin{abstract}
In this paper we do follow a non-stationary discrete approach to model the baseline rate in Brazil. The paper innovates in approaching the decisions in terms of a hike, maintenance or cut in the baseline rate rather than assume that it follows a continuous path. Our model seems to be parsimonious and forecast correctly $83 \%$ of the actual Copom decisions. The estimated parameters define a threshold to the Copom actions which is asymmetric, but finds no significant differences between high and low thresholds.
\end{abstract}

Palavras-chave: Monetary Policy; Taylor Rule; Nonstationary Discrete Approach.

JEL classification: C25, E52, E58

\footnotetext{
* Programa de Pós-Graduação em Economia/UFV. E-mail: sidney.caetano@ufv.br

† Programa de Pós-Graduação em Economia/UFSCar, Campi Sorocaba-SP. E-mail: gedmundos@yahoo.com.br

‡ Curso de Mestrado em Economia Aplicada/UFJF. E-mail: wlrotatori@gmail.com.
} 


\section{Introdução}

Desde os finais dos anos 40, o monetarismo de Milton Friedman já propunha uma regra para a autoridade monetária ao defender a ideia de que esta regra deveria ter como meta a taxa de crescimento de um agregado monetário, taxa que deveria ser sempre constante e igual à taxa de crescimento real estimada. Sua crítica e proposta se orientam contra a condução discricionária da política monetária, uma vez que regras impõem disciplina ao governo, dando maior credibilidade à sua política.

No entanto, as propostas de utilização dos agregados monetários perderam força à medida que mudanças institucionais no mercado financeiro tornaram a velocidade de circulação dos diferentes agregados monetários volátil e, consuentemente, com maior imprevisibilidade do que antes.

Por conseguinte, a simplicidade da regra de Friedman foi substituída por importantes inovações metodológicas e empíricas que alavancaram o debate regras versus discrição em política monetária como um importante campo de pesquisa nos anos 1980 e 1990. Dentro do contexto deste debate, pode-se ressaltar o colapso das regras monetárias baseadas em âncoras nominais, como o regime da taxa de câmbio fixo, ou mesmo crawling peg, nos anos 1990, e sua substituição em termos de condução da política monetária pelo regime de metas de inflação, que se tornou amplamente aceito tanto pelos macroeconomistas teóricos quanto pelos formuladores de política monetária. Regime esse que foi formalmente adotado pela Nova Zelândia em 1989/1990 e, atualmente, é seguido por mais de 20 países - entre desenvolvidos e emergentes - dentre eles o Brasil, desde julho de 1999, após o abandono do regime de âncora cambial.

No Brasil, a condução da política monetária tem sido pautada no "método dos dois alvos" de contenção da inflação, predeterminação da inflação e da taxa Selic, como chama Cysne (2005). Neste regime, uma tendência cada vez mais dominante da política monetária tem sido a adoção da regra de Taylor (1993), que se propõe ser um guia útil para política monetária, além de proporcionar maior transparência e credibilidade.

Desde o início dos anos 90, o uso destas regras de política monetária para avaliar e descrever as ações do banco central tem crescido rapidamente, apoiado por analistas de mercados financeiros, acadêmicos, bancos centrais etc.

No começo, grande parte dos estudos era focado em economias desenvolvidas, como a regra de política monetária para a economia dos Estados Unidos, proposta por Taylor (1993), conhecida atualmente como "Regra de Taylor". Posteriormente, o próprio Taylor (2000) afirmou que ela poderia ser aplicada em economias emergentes.

A maior parte das pesquisas sobre regras de política tem dado ênfase à taxa nominal de juros de curto prazo como principal instrumento, enquanto outras pesquisas, em menor escala, sugerem ou analisam a base monetária ou outro agregado monetário, produto nominal etc. Cabe destacar que Taylor (1979) iniciou seus estudos sobre regras de política usando como instrumento a oferta monetária. McCallum (1988) também enfatiza as vantagens das regras de política que utilizam como instrumento um agregado monetário. No entanto, este instrumento foi perdendo espaço para a taxa de juros em grande parte pelos mesmos motivos que levaram ao declínio no uso de agregados monetários no caso da regra proposta por Friedman. 
No caso de aplicações empíricas para o Brasil, Carneiro \& Wu (2004) destacam que a regra de Taylor tem tido um surpreendente poder explicativo para períodos com políticas monetárias bem diversas, e este poder cresce quando se introduz inércia via alguma hipótese de suavização. Não obstante a utilização da regra de Taylor como forma de determinação do comportamento dos juros possa ter um poder de explicação não desprezível, frequentemente a decisão de interesse para os agentes econômicos toma a forma de uma escolha discreta, ou seja, se haverá um aumento, manutenção ou queda na taxa de juros.

As decisões do Comitê de Política Monetária (Copom) sobre a taxa de juros Selic têm sido acompanhadas por diversas instituições e analistas financeiros, agentes econômicos, acadêmicos, políticos etc. com o intuito de entender e prever possíveis movimentos na taxa de juros. O Copom é o órgão decisório da política monetária do Banco Central do Brasil e o responsável por definir a meta para a taxa Selic, tendo sido instituído em 20 de junho de 1996.

Desde 1996, o Regulamento do Copom sofreu uma série de alterações no que se refere ao seu objetivo, à periodicidade das reuniões, à composição e às atribuições e competências de seus integrantes, visando não apenas a aperfeiçoar o processo decisório no âmbito do Comitê, como também a refletir as mudanças do regime monetário.

Destaca-se entre essas mudanças a adoção em junho de 1999 da sistemática de "metas para a inflação"como diretriz de política monetária. Desde então, as decisões do Copom passaram a ter como objetivo cumprir as metas para a inflação definidas pelo Conselho Monetário Nacional (CMN). Neste regime, se as metas não forem atingidas, cabe ao presidente do Banco Central encaminhar uma Carta Aberta ao Ministro de Estado da Fazenda explicando os motivos do descumprimento, bem como as providências e prazo para o retorno da taxa de inflação aos limites estabelecidos.

Formalmente, os objetivos do Copom são programar a política monetária, definir a meta da taxa Selic e seu eventual viés e analisar o "Relatório de Inflação". A taxa de juros fixada na reunião do Copom é a meta para a taxa Selic (taxa média dos financiamentos diários, com lastro em títulos federais, apurados no Sistema Especial de Liquidação e Custódia), que pode vigorar por todo o período entre reuniões ordinárias do Comitê.

A evolução da taxa de juros no Brasil tem apresentado momentos distintos no decorrer de alguns anos. Algumas das decisões da autoridade monetária, de alta gradual, têm sido implementadas para acomodar o ritmo de expansão da demanda agregada e pela resistência da inflação à queda, tendo em vista a necessidade de assegurar a convergência da inflação para as metas estipuladas pelo Conselho Monetário Nacional (CMN).

Tais decisões de política monetária envolvendo escolhas discretas são muito comuns na prática. Em muitos casos, trata-se de uma escolha entre apenas duas alternativas possíveis, por exemplo, o Copom deve ou não aumentar a taxa de juros Selic meta? Em outros casos, as decisões envolvem escolhas entre múltiplas alternativas possíveis: o Copom deve aumentar, reduzir ou manter inalterada a meta para a taxa Selic?

Analisar os determinantes do processo de escolha entre um número finito de alternativas, a fim de tentar prever o movimento a ser feito pelo Copom sob certas condições, se tornou relevante diante do nervosismo que alguns agentes econômicos têm demonstrado nas vésperas das reuniões que decidem sobre a taxa Selic-meta. 
Dada a relevância, muitos estudos buscam evidências empíricas que indiquem qual deve ser o comportamento da taxa de juros. Entretanto, a maioria tem buscado estimar uma função de reação estruturada com a premissa de uma taxa de juros como variável dependente contínua. Em particular, para o caso brasileiro, podem ser destacados: Figueiredo \& Ferreira (2002), Minella et al. (2002), Favero \& Giavazzi (2002), Carneiro \& Wu (2004), Mendonça (2007) e Modenesi (2008). Todos eles compartilhando a característica comum da continuidade da variável resposta, ou seja, a função de reação do Banco Central ou a regra de política monetária parte do pressuposto de que a taxa de juros é contínua. Não obstante os resultados presentes na literatura indiquem um poder explicativo para tais funções estimadas, esta metodologia não pode ser aplicada para prever os movimentos que representam reversão de tendência com a mesma propriedade, em virtude de suposições relacionadas à estacionariedade e à continuidade da variável resposta.

Diante do exposto, a literatura empírica tem sugerido um enfoque alternativo para a dinâmica de variáveis macroeconômicas em que são verificadas tanto a presença da não estacionariedade quanto da não continuidade. Tal abordagem foi proposta por Hu \& Phillips (2004a), que propuseram uma abordagem de escolha discreta não estacionária para modelar o comportamento empírico do Federal Reserve quanto às mudanças na "Federal Funds Target Rate".

A motivação dos autores é que uma mudança brusca no movimento, mesmo que represente uma reversão de tendência, seja prevista empiricamente. Para tal, suposições sobre a não estacionariedade de covariáveis seriam fundamentais.

No Brasil, alterações bruscas da taxa Selic-meta superiores a 100 pontos base (p.b.), para cima ou para baixo, dificultam ou até mesmo inviabilizam o uso de qualquer modelo contínuo estacionário. Visando a contornar este problema, o presente trabalho considerou a abordagem metodológica proposta por $\mathrm{Hu} \&$ Phillips (2004a).

Para o caso brasileiro, em específico, uma abordagem diferenciada considerando variáveis discretas para o problema de tomada de decisão pelo Banco Central encontra-se em Teles \& Brundo (2006). Estes autores propõem um modelo de escolha discreta, estimado via abordagem bayesiana, onde a construção de três índices de política monetária é proposta, para então estimarem as funções de reação do Banco Central. Os índices são construídos de maneira a representarem as decisões de política. No primeiro índice considera-se apenas o controle da inflação; no segundo índice apenas as variações da Selic que foram motivadas pelo combate a um choque na inflação corrente observada; e no terceiro índice variações motivadas por alterações na expectativa de inflação.

Entretanto, o presente estudo diferencia-se desta abordagem fundamentalmente por não nos restringirmos especificamente à utilização de uma função de reação do Banco Central a variações na inflação e no hiato do produto. Diferentemente, o objetivo é a modelagem da tomada de decisão do Copom relativa à alta, baixa ou manutenção da taxa Selic meta, ou seja, comparativamente apenas restrito ao primeiro índice dos autores supracitados que consideram que todas as variações na taxa Selic meta se devem ao controle da inflação. Ainda, utiliza-se uma abordagem clássica. Não obstante este foco nas variações da taxa enquanto controle da inflação, no presente estudo, por outro lado, são propostos dois limiares endógenos para os cortes de alta e 
baixa na taxa de juros relativamente à taxa de juros latente que é proposta pelo modelo; possibilitando, portanto, a investigação de assimetria na tomada de decisão do Copom relativo à decisão de altas e baixas na taxa Selic, característica essa não analisada por Teles \& Brundo (2006) e não explorada na literatura para a economia brasileira.

Por fim, propõe-se como exercício de adequação do modelo a sua capacidade de antecipar os movimentos do Copom relativos à tomada de decisão de alta, baixa ou manutenção da taxa diferentemente do proposto em Teles \& Brundo (2006), onde os autores concentram sua atenção na estimação de parâmetros variáveis para identificar alterações no comportamento do Banco Central. Em termos de condução da política monetária, a possibilidade de antecipação das decisões do Copom possui efeitos importantes na diminuição do ruído nos mercados financeiros quando da divulgação da decisão do comitê o que ressalta a importância e originalidade do exercício proposto, na medida em que a especificação do modelo é discutida a partir da capacidade de prever os movimentos discretos da autoridade monetária, ou seja, a ênfase não está apenas na investigação de como o Copom reage com os juros a variações na inflação e no hiato do produto como definido por uma regra de juros teórica $a$ priori impondo uma restrição na forma funcional, mas também na capacidade do modelo em se adequar ao comportamento discreto da reação da autoridade monetária considerando um espectro mais amplo de variáveis que compõem a forma funcional do modelo.

Em dois modelos alternativos identificados neste estudo, diferentes daqueles estruturados na literatura empírica brasileira, foram considerado um conjunto de covariáveis relacionadas a uma regra de Taylor e ao processo decisório do Copom, a saber: desvio das expectativas de inflação 12 meses à frente em relação à meta para inflação anual (IPCA \%), hiato do produto, risco país, câmbio nominal, dívida líquida/PIB (em \%), superávit primário do setor público/PIB (em \%), taxa de desemprego, emprego formal, utilização da capacidade de produção da indústria (CNI), oferta monetária-M2/PIB (em \%), índice de confiança do consumidor (ICC); e taxa Selic-meta defasada. Os resultados revelaram dois modelos parcimoniosos apresentando percentuais de acertos consideráveis. A partir destes percentuais, concluiu-se que a taxa de juros latente indicaria uma maior queda do que as estabelecidas pelo Copom, revelando um grau de cautela das autoridades monetárias e de preocupação não somente com a inflação, mas também com o emprego, além de indicar uma preferência pela suavização dos ajustes na taxa em detrimento de movimentos mais abruptos na mesma.

Para tais objetivos, o artigo é organizado na seguinte sequência, além da presente introdução; Seção 2, que faz breves comentários sobre regras de política monetária; Seção 3, que descreve a abordagem discreta aplicada; Seção 4, que apresenta os dados juntamente com os resultados e discussão; e a Seção 5, que finaliza com as conclusões.

\section{Regras de política monetária}

A proposição de uma regra de conduta da política monetária remontaria, segundo Barro (2006), às contribuições seminais de Milton Friedman, já em fins dos anos quarenta. Dentre as regras propostas e implementadas, a destacável tem sido a regra de Taylor, proposta em Taylor (1993) e especificada como: 


$$
i_{t}=\pi_{t}+r^{*}+0.5\left(\pi_{t}-\pi^{*}\right)+0.5\left(y_{t}\right)
$$

em que $i$ é a taxa de juros nominal de curto prazo (taxas dos Federal Funds americanos); $\pi$ é a taxa de inflação medida pelo deflator do PIB; $\pi *$ é a meta de inflação; $r *$ é a taxa real de juros de equilíbrio; e $y$ é o desvio do produto real em relação ao produto potencial.

Um fato interessante da regra, que a diferencia das atuais funções de reação, é ela não ter os parâmetros estimados econometricamente. Foi assumido pelo autor pesos às covariáveis, hiato do produto e desvio da inflação em relação à meta, ambos em 0.5 . Além disto, o autor assumiu um valor de $2 \%$, tanto para a meta de inflação quanto para a taxa de juros de equilíbrio.

Uma crítica a este tipo de enfoque seria que as autoridades monetárias não deveriam usar as regras de forma mecânica. Mas a literatura não tem destacado tal fato, ou seja, não apresentaria propostas que enfatizariam que tal comportamento mecânico deveria ser seguido pelas autoridades monetárias que optassem por uma regra de política. No entanto, as regras de política, como apresentadas na equação (1), não deixariam de ser uma equação matemática. Mas isto não implicaria que se deveria usá-la mecanicamente. Pelo contrário, a maior parte das propostas sobre regras de política monetária sugere que as regras deveriam ser utilizadas como guias de política, inclusive para países emergentes, como pode ser visto em (Taylor 2000, p.502).

Outro fato interessante seria que a aplicação prática da regra requereria certa discricionariedade limitada, uma vez que, em certas ocasiões, exigiria a necessidade de modificação do instrumento de política monetária, devido a algum fator não incluído na regra.

A partir da regra de Taylor, diversos outros estudos procuraram analisar se esta regra, ou uma modificação dela, poderia ser um guia útil em outras economias desenvolvidas e emergentes. Assim, outras funções de reação foram surgindo, muitas delas inserindo outras variáveis na regra de Taylor original e estimando econometricamente seus parâmetros.

Clarida et al. (2000) propuseram a seguinte regra ${ }^{1}$ :

$$
r_{t}^{*}=\alpha+\beta\left[E\left(\pi_{t, k} \mid \Omega_{t}\right)-\pi^{*}\right]+\gamma \mathrm{E}\left(x_{t, k} \mid \Omega_{t}\right)
$$

em que $r *$ é a taxa de juros nominal meta determinada pelo banco central; $\pi$ é a taxa de inflação; $\pi^{*}$ é a meta de inflação; $x$ é o hiato do produto; $E$ é o operador de expectativas condicional; e $\Omega$ é o conjunto de informação disponível em $t$.

Os autores destacaram que, devido à existência de suavização nas taxas de juros, poderia ser difícil encontrar $r *$. Mas a taxa de juros nominal efetiva poderia ser trabalhada como:

$$
i_{t}=(1-\rho) r_{t}^{*}+\rho i_{t-1}+v_{t},
$$

em que: $\rho \in[0,1]$ é o grau de suavização da taxa de juros; $v$ são os choque externos com média zero; e $r *$ é a taxa de juros nominal meta, determinada pela equação (2).

McCallum (2000), diferentemente de outros autores, sugeriu um instrumento alternativo e representou uma regra monetária da seguinte forma:

$$
\Delta b_{t}=\Delta x^{*}-\Delta v_{t}^{a}+0.5\left(\Delta x^{*}-\Delta x_{t-1}\right)
$$

\footnotetext{
${ }^{1}$ A equação (2) seguiu sua apresentação pelos autores Salgado et al. (2005), pois se acredita que ela tenha ficado mais fácil para o leitor entender do que a própria apresentação de Clarida et al. (2000).
} 
em que $\Delta b$ é a variação da base monetária em logaritmo; $\Delta x *$ é a meta do crescimento do PIB nominal; $\Delta v^{a}$ é a taxa de crescimento médio da velocidade da base monetária (últimos 16 trimestres); e $\left(\Delta x^{*}-\Delta x_{t-1}\right)=$ refletiria as variações de longo prazo na demanda por base monetária.

Outras regras também foram investigadas e comparadas (McCallum 2000, p. 7). Salgado et al. (2005) propuseram a seguinte função de reação:

$$
i_{t}=\alpha+\beta i_{t-1}+\delta \pi_{t}+\kappa \tilde{y}_{t}+\theta \Delta R_{t}
$$

A função de reação (5) apenas incluiria a variação percentual nas reservas internacionais além das tradicionais como covariáveis na função de reação. Os autores procuraram estimar esta função de reação para o Banco Central do Brasil durante o Plano Real (08/1994 a 12/2000), modelada via modelo TAR (Limiar Autorregressivo) para captar as mudanças de regime na determinação da taxa nominal de juros. Segundo os autores, o desempenho do modelo nãolinear foi significativamente melhor do que o de uma regra de Taylor ajustada às taxas de juros brasileiras.

Carneiro \& Wu (2004), entre seus objetivos, procuraram analisar o condicionamento dos juros internos às necessidades de financiamento externo, o que implicaria a subordinação da taxa de juros a alguma variável que captasse as pressões de choques externos sobre o Balanço de Pagamentos e se manifestasse diante de ameaças externas em direção a uma fuga de capitais. Os autores estimaram dois modelos, também para o período do Plano Real (1994:04 a 2000:02). O primeiro, representado pela equação (6), seria uma regra de Taylor tradicional com "suavização". Segundo a regra, a taxa de juros nominal reagiria a choques de oferta, tais como surpresas na taxa de inflação e desvio do nível de atividade de seu potencial.

$$
i_{t}=(1-\lambda) i_{t-1}+\lambda\left[\gamma_{1}\left(\pi_{t}-\pi_{t}^{e}\right)+\gamma_{2} h_{t-1}+\gamma_{3}\right]+\varepsilon_{t}
$$

em que $i$ é a taxa de juros Selic efetiva no trimestre; $\pi$ é a taxa de inflação medida pelo IPCA acumulada no trimestre; $\pi^{e}$ é a taxa de inflação esperada; $h$ é o hiato do PIB trimestral; e $\varepsilon$ é um choque tal que $\varepsilon_{t}=v_{t}+\theta_{1} v_{t-1}+\theta_{2} v_{t-2}$, $\operatorname{com} v_{t} \sim N(0, \sigma)^{2}$.

Já o segundo modelo, estimado pela ampliação da equação (6), apenas se acrescentaria o nível das reservas internacionais como variável explicativa. Nota-se forte semelhança com a função de reação proposta em Salgado et al. (2005), representada na equação (5).

$$
i_{t}=(1-\lambda) i_{t-1}+\lambda\left[\gamma_{1}\left(\pi_{t}-\pi_{t}^{e}\right)+\gamma_{2} h_{t-1}+\gamma_{3} r_{r}+\gamma_{4}\right]+\varepsilon_{t} .
$$

Na equação (7), $r$ seria a média trimestral do estoque mensal de reservas internacionais, conceito de liquidez internacional, final de período.

Os trabalhos supracitados estiveram centrados na análise da taxa de juros como uma variável dependente contínua, em que a função de reação do Banco Central ou a regra de política monetária partem do pressuposto de que a taxa de juros é contínua. Neste sentido, a próxima seção apresenta uma abordagem alternativa, que será aplicada neste trabalho.

\footnotetext{
${ }^{2}$ Segundo os autores, caso a autocorrelação não fosse considerada, os coeficientes estimados continuariam não viesados, mas alguns se tornariam insignificantes.
} 


\section{Abordagem discreta}

A literatura de regras monetária aponta que a regra de Taylor tem sido estimada com as covariáveis padrão gerando a possibilidade de inserir variáveis adicionais como a taxa de desemprego, oferta monetária, índices relacionados ao crédito e confiança dos consumidores, entre outros.

Em função da complexidade do processo decisório do Copom, dado que seus membros preveem os riscos em torno da convergência da inflação para as metas estipuladas pelo $\mathrm{CMN}$, diversas informações importantes têm sido consideradas no "Relatório de Inflação", e não somente variáveis tradicionais como o hiato de produto e os desvios da inflação (expectativas da inflação) em relação à meta. Portanto, seria fundamental verificar se algumas destas variáveis também influenciariam a direção do movimento das taxas de juros Selic-meta: alta, baixa ou estabilidade.

A vantagem de um modelo probit multinomial (MPM) é que, ao contrário do modelo probit tradicional baseado em apenas duas alternativas, o multinomial possibilitaria análise de mais que duas alternativas.

Para Maddala \& Kim (1999), a desvantagem, ou limitação do MPM, seria que para $\mathrm{n}$ alternativas, o cálculo das probabilidades envolveria integrais de dimensão $(n-1)$ e assim, dois métodos alternativos seriam operacionalizáveis: (i) o método de Monte Carlo que estipularia os vetores dos resíduos a partir de uma distribuição normal multivariada; e (ii) o método de aproximação numérica que apresentaria resultados consistentes para o caso em que as variáveis fossem não negativamente correlacionadas e as variâncias iguais.

Cameron \& Trivedi (2005) apontaram que os parâmetros da regressão e das variâncias dos resíduos seriam obtidas por máxima verossimilhança. Entretanto, não haveria uma expressão definitiva para as probabilidades das alternativas consideradas.

Em um modelo mais amplo, portanto, quatro métodos seriam apresentados como alternativos na literatura: (i) o método de simulação das estruturas de máxima verossimilhança; (ii) o método dos momentos simulados; (iii) o método dos escores simulados; e (iv) os métodos bayesianos.

As alternativas quanto à escolha dos métodos e dos modelos MPM basearse-iam nas especificações da matriz de covariância, e as restrições na mesma poderiam assegurar a identificação do modelo. Entretanto, uma importante questão surgida foi a de trabalhar com séries de tempo estacionárias e/ou nãoestacionárias em abordagens discretas. Fato relevante, principalmente, nesta aplicação, pois muitas das séries econômicas relatadas pelo Copom como importantes no cenário econômico implícito na sua decisão sobre os juros podem apresentar comportamento não-estacionário.

A raiz da problematização, combinação de escolha discreta com covariáveis integradas, foi devida a Park \& Phillips (2000), em que uma teoria assintótica se fazia necessária para tais modelos. Embora seja reconhecida a consistência de estimativas de máxima verossimilhança (ML) para tais processos, o vetor de parâmetros estimados seria uma combinação de duas componentes vinculadas à direção do verdadeiro vetor de parâmetros, ora seguindo uma direção paralela, ora seguindo uma direção ortogonal. Assim, os testes de escore, baseados na segunda derivada das funções de máxima verossimilhança, não seriam robustos em função de as amostras convergirem para dois pontos diferentes, os quais, portanto, seguiriam uma distribuição do tipo arco seno. 
Como a matriz hessiana converge fracamente para a solução proposta por Park \& Phillips (2000), foi elaborado um índice que representasse a combinação de soluções decorrentes da convergência para os dois pontos distintos.

Tomando-se como suporte a modelagem empírica de problemas com covariávies não-estacionárias, Hu \& Phillips (2004b) apresentaram um modelo de escolha discreta não-estacionário derivado dos problemas apresentados por Park \& Phillips (2000). Posteriormente, Phillips et al. (2007) fizeram correções em Hu \& Phillips (2004b): "We correct the limit theory presented in an earlier paper by $\mathrm{Hu}$ and Phillips (Journal of Econometrics, 2004a) for nonstationary time series discrete choice models with multiple choices and thresholds." Em seguida, Hu \& Phillips (2004a) propuseram uma aplicação de tal abordagem discreta não estacionária para a taxa de juros americana, conforme já comentado.

O modelo considerado neste trabalho se baseia nesta possibilidade de escolhas multivariadas com séries não estacionárias, em que uma mesma estrutura de modelo pode ser desenvolvida para a economia brasileira, permitindo investigar a possibilidade de o Copom elevar, reduzir ou manter as taxas de juros.

Assim, a estrutura de um modelo com covariáveis integradas seria:

$$
\begin{aligned}
& r_{t}^{*}=x_{t}^{\prime} \beta_{0}-\varepsilon_{t}, \quad \text { para } t=1, \ldots, n . \\
& y_{t}^{*}=r_{t}^{*}-r_{t-1},
\end{aligned}
$$

em que $r_{t}^{*}$ é a verdadeira mas não observável taxa meta ótima; $x_{t}$ é uma matriz que representa as variáveis explicativas que podem ser $I(0)$ ou $I(1)$ ou uma mistura destes; e $\epsilon_{t}$ é o vetor de erros considerado ser i.i.d. com função distribuição $F$; e $r$ é a taxa Selic-meta estabelecida no encontros do Copom. A variável latente $y_{t}^{*}$ mensura o desvio entre a taxa meta ótima, $r_{t}^{*}$, e a taxa meta estabelecida em um encontro anterior, $r_{t-1}$.

Como as variáveis $r_{t}^{*}$ e $y_{t}^{*}$ não são observadas, utiliza-se uma especificação "triple-choice" para o modelo de escolha discreta em que $y_{t}=-1$ implica queda na taxa meta, $y_{t}=0$ denota estabilidade e $y_{t}=1$ significa um aumento. Ou seja,

$$
\begin{array}{lrl}
y_{t}=-1 & \text { se } y_{t}^{*}<\mu_{n 0}^{1} \\
y_{t}=0 & \text { se } \mu_{n 0}^{1} \leq y_{t}^{*} \leq \mu_{n 0}^{2} \\
y_{t}=1 & \text { se } y_{t}^{*}>\mu_{n 0}^{2}
\end{array}
$$

em que $\mu_{n 0}^{1}$ e $\mu_{n 0}^{2}$ são os parâmetros limiares ${ }^{3}$.

A taxa Selic-meta anunciada no tempo $t$ é especificada da seguinte maneira:

$$
\begin{array}{ll}
r_{t}=r_{t-1}-\Delta_{t} & \text { se } y_{t}=-1 \\
r_{t}=r_{t-1} & \text { se } y_{t}=0 \\
r_{t}=r_{t-1}+\Delta_{t} & \text { se } y_{t}=1
\end{array}
$$

Neste sentido, as equações (8), (9), (10) e (11) constituem o modelo base.

\footnotetext{
${ }^{3}$ A estimação dos parâmetros limiares se diferencia da proposta em Teles \& Brundo (2006), na medida em que para estes autores existe apenas um parâmetro limiar, $\gamma$, que é estimado na formulação deles; enquanto que a proposta neste trabalho compõe-se de dois parâmetros que permitem uma análise assimétrica para decisões de alta e baixa.
} 
Generalizando o modelo, assume-se que a variável dependente $y_{t}^{*}$ seja nãoobservada, mas o indicador $y_{t}$ pode ser. Assim,

$$
\begin{aligned}
& y_{t}=0 \text { se } y_{t}^{*} \in\left(-\infty, \sqrt{n} \mu_{0}^{1}\right) \\
& y_{t}=1 \text { se } y_{t}^{*} \in\left(\sqrt{n} \mu_{0}^{1}, \sqrt{n} \mu_{0}^{2}\right) \\
& \vdots \\
& y_{t}=J-1 \text { se } y_{t}^{*} \in\left(\sqrt{n} \mu_{0}^{J-1}, \sqrt{n} \mu_{0}^{J}\right) \\
& y_{t}=J \text { se } y_{t}^{*} \in\left(\sqrt{n} \mu_{0}^{J}, \infty\right)
\end{aligned}
$$

Pressupõe-se que $x_{t}$ seja predeterminado e séries de tempo integradas $x_{t}=$ $x_{t-1}+v_{t} \operatorname{com} x_{0}=O_{p}(1)$ e $v_{t}=\prod(L) e_{t}=\sum_{i=1}^{\infty} \prod_{i} e_{t-i}$ em que os coeficientes $\Pi i$, as inovações i.i.d. $e_{t}$ e $F$ satisfazem certas condições de regularidade ${ }^{4}$. Em (12), os parâmetros threshold seriam $\mu_{n 0}^{j}=\sqrt{n} \mu_{0}^{j}$, de acordo com a ordem estocástica do indicador $y_{t}^{*}$ para tamanho de amostra $t=O(n)$.

Um modelo de escolha discreta geral, com distribuição de probabilidade condicional de $y t$, escrita como $P\left(y_{t}=j \mid \operatorname{Im}_{t-1}\right)=P_{j}\left(x_{t} ; \theta_{0}\right)$, teria a forma:

$$
\begin{aligned}
& P_{0}\left(x_{t} ; \theta_{0}\right)=1-F\left(x_{t}^{\prime} \beta_{0}-\sqrt{n} \mu_{0}^{1}\right) \\
& P_{j}\left(x_{t} ; \theta_{0}\right)=F\left(x_{t}^{\prime} \beta_{0}-\sqrt{n} \mu_{0}^{j}\right)-F\left(x_{t}^{\prime} \beta_{0}-\sqrt{n} \mu_{0}^{j+1}\right) \quad j=1, \ldots, J-1 \\
& P_{J}\left(x_{t} ; \theta_{0}\right)=F\left(x_{t}^{\prime} \beta_{0}-\sqrt{n} \mu_{0}^{J}\right) .
\end{aligned}
$$

Logo, a expectativa condicional de $y_{t}$ seria:

$$
m\left(x_{t} ; \theta_{0}\right)=\sum_{j=0}^{J} j P_{j}\left(x_{t} ; \theta_{0}\right)=\sum_{j=1}^{J} F\left(x_{t}^{\prime} \beta_{0}-\sqrt{n} \mu_{0}^{j}\right) .
$$

Seja a função indicadora:

$$
\Lambda(t, j)=\frac{\prod_{i=0, \ldots, J \text { e } i \neq j}\left(y_{t}-i\right)}{\prod_{i=0, \ldots, J \text { e } i \neq j}(j-i)} .
$$

É fácil verificar que $\Lambda(t, j)=I\left(y_{t}=j\right)$ seria a função indicadora para $y_{t}=j$. Assim, o logaritmo da função de verossimilhança pode ser escrito como:

$$
\log L_{n}(\theta)=\sum_{t=1}^{n} \sum_{j=0}^{J} \Lambda(t, j) \log P_{j}\left(x_{t} ; \theta\right) .
$$

Nota-se que $P_{j}\left(x_{t} ; \theta_{0}\right)$ envolve uma função não-linear $F\left(x_{t}^{\prime} \beta_{0}-\sqrt{n} \mu_{0}^{j}\right)$ para um processo $x_{t} I(1)$. Os parâmetros $\beta$ e $\mu$ podem ser estimados tanto por uma regressão probit quanto logit. No presente caso, utiliza-se a primeira.

\section{Resultados e discussão}

\subsection{Os dados}

Sabe-se que, na prática, o Copom ajusta a taxa de juros Selic-meta de forma discreta, ou seja, nas datas de reuniões agendadas antecipadamente. Algumas

\footnotetext{
${ }^{4}$ Ver Hu \& Phillips (2004a).
} 
alterações podem ser realizadas de acordo com o entendimento do Comitê, mas de qualquer forma não ocorre de forma contínua. Tais reuniões são importantes e, por isso, aguardadas tanto pelos analistas de mercado, quanto pelos acadêmicos, governo e mídia, pois a taxa de juros definida é referência no mercado para as demais taxas de juros.

As reuniões ordinárias do Copom dividem-se em dois dias: a primeira sessão às terças-feiras e a segunda às quartas-feiras com periodicidade mensal a partir de 2000, tendo sofrido uma redução no número de reuniões ordinárias para oito ao ano a partir de 2006, sendo o calendário anual divulgado até o fim de outubro do ano anterior.

A amostra compreende os meses de janeiro de 2002 a dezembro de $2008^{5}$. Dentro deste período, ocorreram 72 reuniões agendadas e 12 reuniões não marcadas, totalizando 84 encontros para decidir a direção e magnitude da taxa de juros básica da economia brasileira, a taxa Selic-meta. Assim sendo, a maior parte das alterações ocorreu nas datas das reuniões estabelecidas pelo Copom, e sua magnitude foi múltipla de 25 pontos base (p.b.). Observa-se na Tabela 1 que o Copom aumentou 30 vezes a taxa Selic, reduziu-a 18 vezes e a manteve estável em 36 encontros.

Entretanto, pode-se notar certa suavização nos movimentos na taxa básica. Em particular, o ciclo de alta implementado a partir de meados de 2002 implicou aumento de 8 pontos percentuais antes de iniciar um novo ciclo de baixa na taxa; enquanto o ciclo de alta iniciado em setembro de 2004 implicou aumento de 4 pontos percentuais, como pode ser observado na Figura 1. Além disso, no período entre setembro de 2005 e setembro de 2009, observa-se uma maior sequência de reduções como revela a Figura 2.

Este gradualismo na política monetária pode ser visto também pela preferência de magnitude quando o Copom decide alterar a taxa Selic, tanto para aumentar quanto para reduzir os juros concentrada em variações de 0,25 e 0,50 pontos percentuais, refletindo ainda alguma incerteza quanto à reação dos agentes frente aos movimentos dos juros.

Tabela 1: Alterações na Taxa Selic Meta

\begin{tabular}{lrrrrrrr}
\hline Magnitude & $-2,50$ & $-2,00$ & $-1,50$ & $-1,00$ & $-0,75$ & $-0,50$ & $-0,25$ \\
Frequência & 1,00 & 1,00 & 2,00 & 2,00 & 3,00 & 12,00 & 9,00 \\
Magnitude & 0,00 & 0,25 & 0,50 & 0,75 & 1,00 & 3,00 & - \\
Frequência & 36,00 & 3,00 & 9,00 & 2,00 & 2,00 & 2,00 & - \\
\hline
\end{tabular}

Dados calculados pelos autores

É relevante que os agentes econômicos percebam a meta ajustada pelo CMN como o objetivo da política monetária para que suas expectativas caminhem na direção da trajetória das metas estabelecidas. Com isso, as metas reduzem a possibilidade de reindexação da economia e de aumento da persistência da inflação.

A imposição de juros significativamente inferiores ao considerado "correto" pelos agentes econômicos poderia ser interpretada como trajetórias de possíveis aumentos futuros de inflação. Por outro lado, juros extremamente

\footnotetext{
${ }^{5} \mathrm{O}$ período inicial do presente estudo não é inferior a 2002 devido à inexistência de informações sobre as expectativas de inflação, variável relevante dentro do atual regime de metas e no presente estudo.
} 


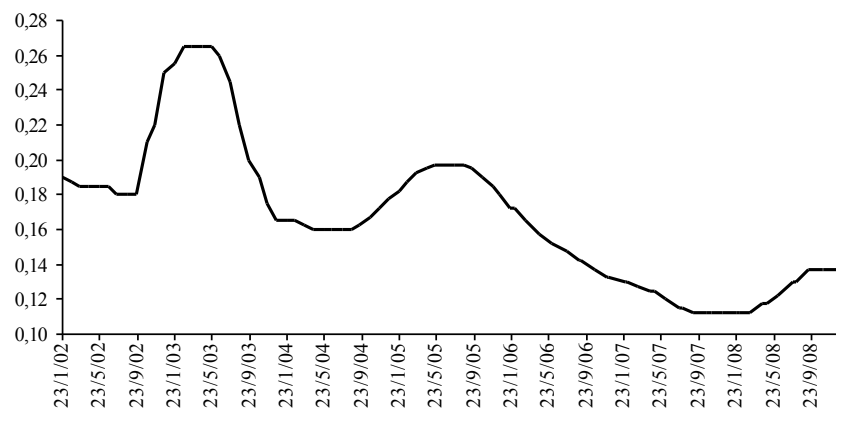

Figura 1: Taxa Selic-Meta

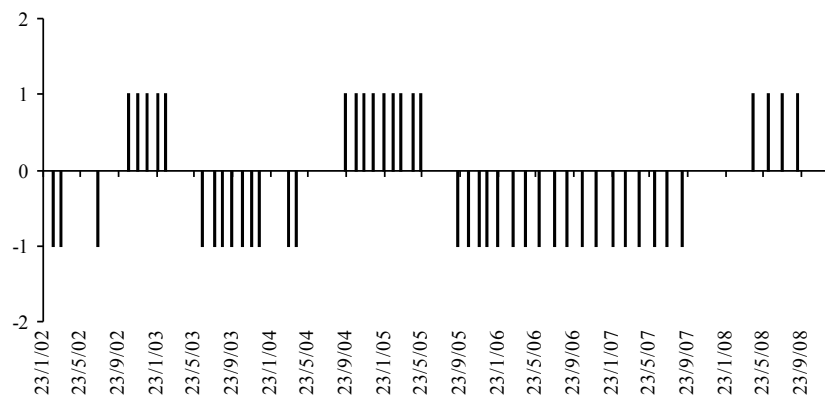

Figura 2: Mudanças discretas na taxa Selic-meta.

superiores podem gerar altos custos em termos de produto e emprego. Uma vez percebido um desvio da inflação, os agentes revisariam rapidamente suas expectativas, podendo colocar em risco o cumprimento das metas para a inflação, o que demonstra certa complexidade.

Além da taxa de juros Selic, a observação de diversas variáveis econômicas mostra-se relevante. Neste sentido, o trabalho utilizou diversas séries de tempo tais como: desvio das expectativas de inflação 12 meses à frente em relação à meta para inflação anual (IPCA \%), hiato do produto, risco país, câmbio nominal, dívida líquida/PIB (em \%), superávit primário do setor público/PIB (em \%), taxa de desemprego, emprego formal, utilização da capacidade de produção da indústria (CNI), oferta monetária-M2/PIB (em \%), índice de confiança do consumidor (ICC) e taxa Selic-meta defasada.

Em seguida, a série observada das taxas de juros meta anunciadas e uma série estimada das taxas de juros latentes foram usadas juntas para captar as intervenções, permitindo inferir sobre o comportamento do Copom. Utilizase uma defasagem de um mês para as variáveis de forma a levar em consideração o tempo defasado de chegada de sua informação. Neste sentido, o modelo está em um formato preditivo.

Nota-se que o presente método adotado em particular envolve uma regra de decisão baseada no desvio entre a regra latente e a taxa Selic-meta defasada, $y_{t}^{*}=r_{t}^{*}-r_{t-1}$, cuja ordem estocástica é relevante dentro do modelo. Ao aplicar o teste de raiz unitária Phillips-Perron (PP), para ambos os desvios, modelos 1 e 2, obtém-se as estatísticas, $-2,76$ e $-2,31$, respectivamente, que não rejeitam a hipótese nula de raiz unitária ao nível de significância de 5\%. Ao aplicar o 
teste PP nas demais séries, obtêm-se diferentes ordens de integração ${ }^{6}$. Ou seja, a presença de algumas séries não estacionárias dentro da atual investigação é verificada, tornando, assim, a aplicação da abordagem de escolha discreta nãoestacionária relevante.

\subsection{Modelos Estimados}

Os resultados finais estimados são apresentados na Tabela 2. Inicialmente, foram utilizadas todas as variáveis explicativas na modelagem, eliminando as variáveis cujos coeficientes não se mostraram estatisticamente significativos a pelo menos $5 \%$ de significância. Tal procedimento gerou o modelo 1 , no qual permaneceram apenas o desvio das expectativas de inflação 12 meses à frente em relação à meta para inflação anual (IPCA \%), o índice de confiança do consumidor e a taxa Selic-meta defasada.

Já o modelo 2 é o resultado de várias combinações de variáveis explicativas visando sempre à sua significância, bem como à maior predição do modelo. Neste sentido, permaneceram apenas o desvio das expectativas de inflação 12 meses à frente em relação à meta para inflação anual (IPCA \%), o superávit primário do setor público/PIB (em \%), o emprego formal, o índice de confiança do consumidor (ICC) e a taxa Selic-meta defasada.

Se for considerado o emprego formal e o índice de confiança do consumidor uma proxy do hiato do produto, uma expressão para a regra de Taylor surgiria, fato não observado no estudo de Hu \& Phillips (2004a).

Os resultados mostram que a taxa Selic-meta é maior (menor) quando a expectativa de inflação futura supera (não supera) a meta para a inflação ou quando a confiança dos consumidores aumenta, o que indicaria uma reação ao aumento da demanda. Ainda, a existência de uma política suavizada para o comportamento dos juros é observada. Já no segundo modelo, a importância do superávit primário e do emprego formal na determinação de intervenção é adicionada, revelando que o superávit primário pode ajudar a política monetária na estabilidade de preços e na possibilidade de redução da taxa de juros básica da economia brasileira. Já o emprego formal pode estar sinalizando uma condição de oferta maior, em que as empresas passam a contratar mais para aumentar sua produção ou para atender a uma projeção mais otimista de aumento de demanda.

Quanto ao parâmetro de corte e alta nos juros, o modelo 1 indicou um parâmetro limítrofe para o corte na taxa Selic-meta de 80 p.b., enquanto para o aumento é de 76 p.b. Já o segundo modelo estima parâmetros de 112 p.b. e 110 p.b. para cortes e aumentos nos juros, respectivamente. Em ambos os modelos, estes parâmetros foram estatisticamente significativos e revelam assimetria nos limiares.

Tal observação revela uma política bem ajustada, em que o direcionamento da política monetária se deveu apenas às variações macroeconômicas, sem intervenção política. A completa assimetria revela, ainda, o correto direcionamento da política monetária e possibilita até mesmo um esboço de uma taxa de juros latente.

\footnotetext{
${ }^{6}$ A estatística $t$ para as demais séries em nível foram: Taxa Selic-Meta $(-1,241)$, EIPCA-META $(-2,535)$, Hiato do Produto $(-3,699)$, Risco País $(-1,528)$, Câmbio Nominal $(-1,284)$, Dívida/PIB (1.074), Superávit/PIB (-1.881), Emprego Formal (2.586), Taxa de Desemprego $(-1,035)$, UCI-CNI $(-3,564)$, M2/PIB $(3,148)$, e ICC $(-2,246)$.
} 
Tabela 2: Modelo de regressão probit e parâmetros threshold

\begin{tabular}{lcr}
\hline Variáveis & Modelo 1 & Modelo 2 \\
\hline$\left(E \pi^{12}-\pi^{*}\right)$ & 0.0135 & 0.0182 \\
& $(0.0038)$ & $(0.0060)$ \\
ICC & 0.0527 & 0.0006 \\
& $(0.0160)$ & $(0.0002)$ \\
$r_{t-1}$ & 0.9281 & 0.7283 \\
$S / P I B$ & $(0.0431)$ & $(0.0964)$ \\
& - & -0.0139 \\
Emprego & - & $(0.0058)$ \\
& & -0.0010 \\
$\mu_{1 n}$ & -0.0080 & $-0.0003)$ \\
& $(0.0022)$ & $(0.0029)$ \\
$\mu_{2 n}$ & 0.0076 & 0.0110 \\
& $(0.0027)$ & $(0.0035)$ \\
\hline
\end{tabular}

Valores calculados pelos autores; desvio padrão entre parênteses.

As Figuras 3 e 5 mostram as taxas de juros latentes geradas pelos modelos 1 e 2 e a taxa Selic-meta anunciada, respectivamente. Já as Figuras 4 e 6 apresentam os desvios das taxas de juros latentes estimadas em relação à taxa de juros Selic-meta defasada para os modelos 1 e 2, respectivamente. Observa-se nas Figuras 3 e 5 que o comportamento da taxa de juros latente é mais volátil, enquanto as Figuras 4 e 6 revelam os limiares e o comportamento dos desvios que induzem ao corte, ao aumento, ou à manutenção na taxa meta.

É interessante notar que a partir das observações dos cortes e reduções na taxa o movimento de alta mais frequente teve magnitude de 50 p.b., enquanto o movimento de baixa mais frequente também teve a mesma magnitude.

Quando se compara com os resultados dos modelos 1 e 2 fica claro, primeiro, que o Copom precisa de uma variação maior do que o mais frequente dos valores no índice para produzir uma queda do que um aumento na taxa. Segundo, em ambos modelos os limiares são significativamente maiores para tomar uma decisão, seja de alta seja de baixa do que as magnitudes observadas de 50 p.b. indicando, portanto, um comportamento conservador do Copom.

Além disso, é possível observar nas Figuras 3 e 5 que o índice em ambos os modelos antecipa em aproximadamente um período os movimentos observados na taxa Selic, o que pode ser confirmado quando se comparam as Figuras A.3 e A.2, no apêndice, que mostram as mudanças na taxa Selic previstas pelos modelos 1 e 2, com a Figura A.1, que mostra as mudanças efetivamente ocorridas na taxa. Este resultado de antecipação dos movimentos na taxa de referência pelos modelos também é observado em Hu \& Phillips (2004a) no exercício para a economia americana, a diferença é que o prazo de antecipação é maior para o caso americano que, de acordo com os autores, se estende até três meses em alguns períodos.

Os modelos são estimados baseando-se em três decisões (aumento, redução, manutenção). Dessa forma, pode-se verificar o poder preditivo dos modelos, comparando as decisões previstas pelos modelos com as decisões observadas, conforme destaca a Tabela 3. De 72 encontros agendados pelo Copom para decidir sobre a taxa Selic-meta observados no período de estudo, o modelo 1 previu corretamente 53 (74\%), enquanto o modelo 2 previu 60 (83\%), o 


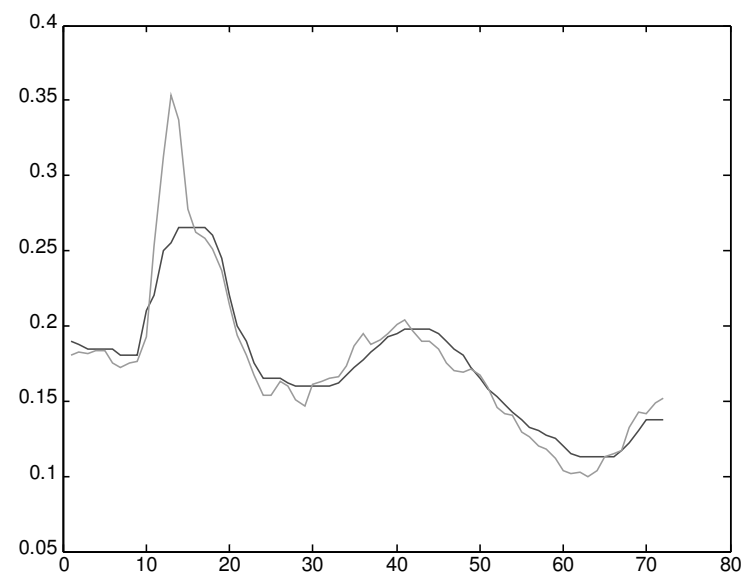

Figura 3: Taxa de juros latente gerada pelo modelo 1 e taxa Selic meta anunciada.

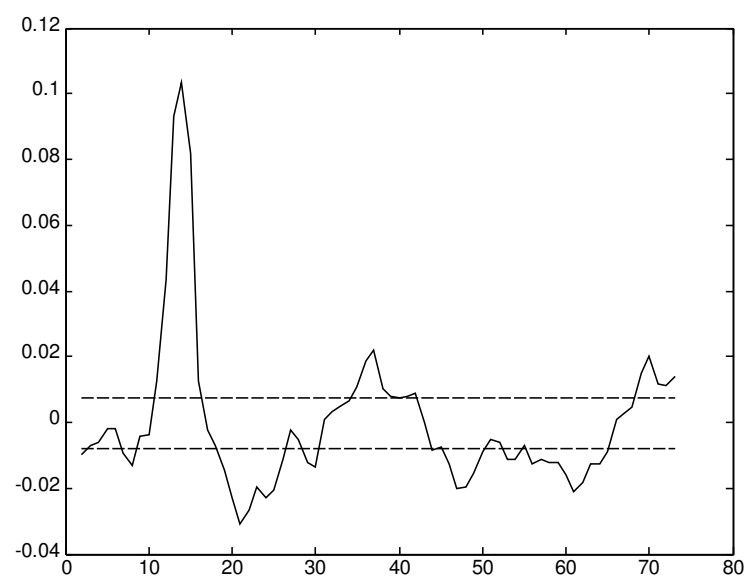

Figura 4: Desvios da taxa de juros latente estimada da taxa de juros Selic meta defasada.

que pode ser interpretado como uma boa capacidade preditiva, considerandose a instabilidade dos dados brasileiros propiciada principalmente pelo período da crise política na transição do governo FHC para o governo Lula.

Ao considerar variáveis importantes como a relação superávit primário do setor público/PIB e emprego formal, é possibilitado um melhor ajuste do processo decisório. Isto vai ao encontro do esforço fiscal estabelecido a partir das metas de superávit fiscal, o que se justifica, pois o superávit primário tende, a longo prazo, amenizar o ônus dos juros sobre a dinâmica dívida interna pública, permitindo, assim, uma menor probabilidade de aumento nos juros, além do objetivo do atual governo de gerar mais emprego formal.

Este resultado de uma maior capacidade preditiva do modelo 2 em relação ao modelo 1 também pode ser verificado observando-se as Figuras A.4 a A.9 no apêndice, que apresentam as probabilidades para corte, aumento e estabilidade da taxa Selic-meta gerada pelos modelos. Neste caso, quando se consi- 


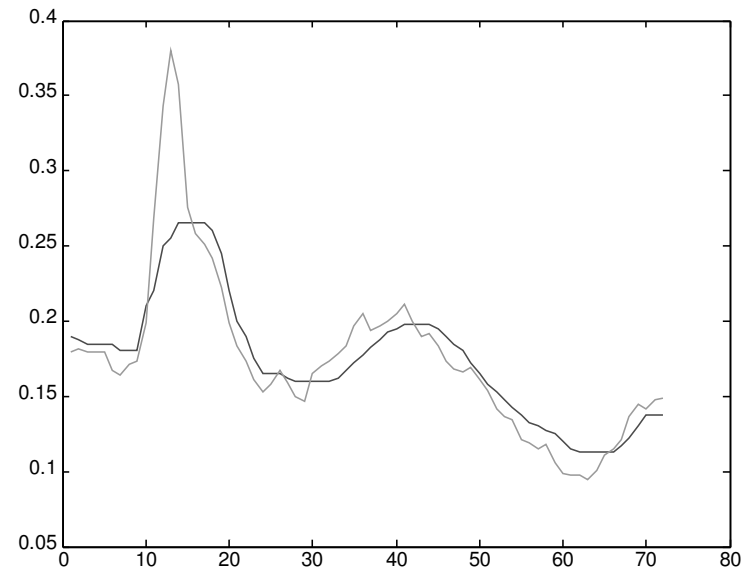

Figura 5: Taxa de juros latente gerada pelo modelo 2 e taxa Selic meta anunciada.

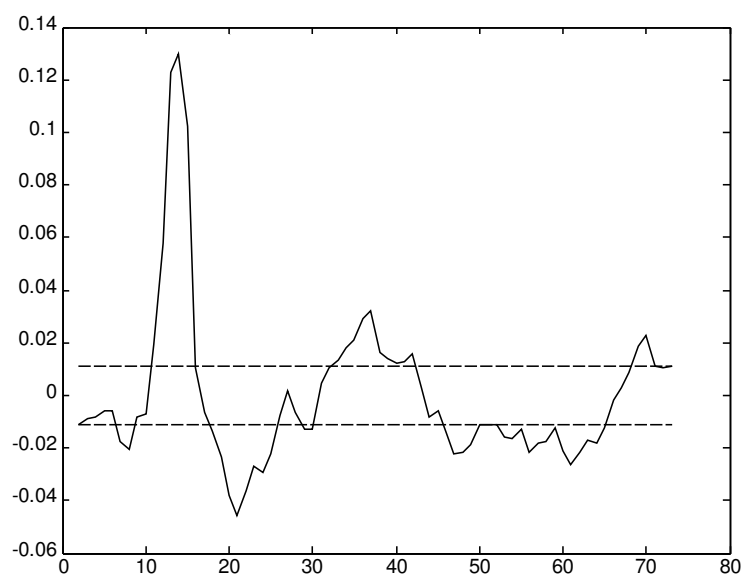

Figura 6: Desvios da taxa latente estimada em relação à taxa de juros Selic-meta(-1).

Tabela 3: Previsões de intervenção política

\begin{tabular}{lccc}
\hline Modelo 1 & Cortes & Estabilidade & Aumento \\
\hline Cortes previstos & 24 & 07 & 00 \\
Estabilidade prevista & 06 & 14 & 03 \\
Aumentos previstos & 00 & 03 & 15 \\
\hline Modelo 2 & Cortes & Estabilidade & Aumento \\
\hline Cortes previstos & 26 & 05 & 00 \\
Estabilidade prevista & 04 & 17 & 01 \\
Aumentos previstos & 00 & 02 & 17 \\
\hline
\end{tabular}


dera o período compreendido entre o terceiro trimestre de 2005 e o terceiro trimestre de 2007 em que o Copom programa um longo período de redução da taxa de juros, o modelo 2 apresenta uma probabilidade de manutenção da taxa (Figura A.8) menor do que a apresentada pelo modelo 1 (Figura A.6), indicando uma melhor adequação do modelo ao cenário de queda relativamente contínua nos juros.

\section{Considerações Finais}

Este trabalho usou uma abordagem de escolha discreta não-estacionária para analisar a dinâmica das intervenções do Copom na taxa de juros básica da economia, a taxa Selic-meta, permitindo, assim, inserir variáveis não estacionárias apresentadas no "Relatório de Inflação" do Banco Central do Brasil. O principal diferencial é justamente o uso da abordagem discreta em contraste com a utilização de uma abordagem contínua para a taxa de juros, o que permite modelar a decisão discreta do Copom em relação à taxa básica. Análises que incluam este resultado são de grande relevância principalmente pelo nervosismo apresentado pelo mercado em períodos próximos às reuniões do Copom.

Foram identificados dois modelos bastante parcimoniosos, sendo o primeiro com as variáveis desvio das expectativas de inflação doze passos à frente em relação à meta, o índice de confiança do consumidor e a taxa de juros defasada, enquanto o segundo modelo acrescenta ao primeiro a razão superávit primário do setor público/PIB e o emprego formal. Os resultados indicam que ambos os modelos empíricos preveem as decisões de intervenção no mercado usando poucas séries econômicas, e o segundo modelo apresenta um maior percentual de acertos em termos globais. Os resultados revelam ainda a relevância de outras variáveis explicativas no processo de decisão do Copom, além das tradicionais variáveis usadas em regras de Taylor.

Os parâmetros estimados para os limiares da tomada de decisão, ou seja, os valores para os quais o índice estimado (taxa de juros latente) quando cruzados indicariam um movimento de queda ou de aumento da taxa de juros se mostraram assimétricos em ambos os modelos. Em particular, os parâmetros para quedas são sempre superiores aos parâmetros de aumento revelando uma preferência pela suavização das ações do Copom quando este considera a necessidade de uma queda na taxa Selic, um resultado em consonância com a literatura a respeito para o Brasil, e com a observação prática das decisões. Este resultado também foi observado por Hu \& Phillips (2004a) quando analisaram as decisões do FED para a economia americana.

Contudo, o mais interessante a ser notado é que para o segundo modelo, que tem melhor capacidade de prever os movimentos, o parâmetro do limiar de corte da taxa Selic foi de 112 p.b., ou seja, mais que o dobro da tomada de decisões mais frequentes do Copom que é de 50 p.b., no período de análise. Ou seja, a taxa de juros latente estaria indicando uma queda muito maior do que aquelas frequentemente anunciadas, revelando o alto grau de "conservadorismo" na tomada de decisões, que pode ser reinterpretado por uma preferência pela suavização dos ajustes na taxa em detrimento de movimentos com maior intensidade. Este último resultado está em concordância com o fato de que a incerteza, presente nas estimativas dos parâmetros, pode reduzir a resposta dos instrumentos de política aos choques na economia, quando 
comparada a um ambiente de certeza, motivando uma suavização na trajetória das taxas de juros como defendem Clarida et al. (2000).

Ao ser observado a não-estacionariedade das covariáveis do superávit primário do setor público/PIB, emprego, ICC, Selic-meta, além dos desvios estimados pelos modelos 1 e 2, conclui-se que, dada a dependência da distribuição assintótica dos desvios da taxa de juros estimada em relação à meta, em ambos os modelos, e, ainda, o fato de as estimativas serem não estacionárias, essas covariáveis garantem uma ordem estocástica unitária. Logo, a decisão das autoridades quanto ao patamar da taxa de juros-meta é uma decisão temporal, embora se considere que uma taxa de juros objetiva possa ser estabelecida.

\section{Agradecimentos}

Os atores agradecem ao $\mathrm{CNPq}$ o apoio financeiro para o desenvolvimento deste estudo, a Ling Hu pela disponibilidade do "Matlab programs" e aos avaliadores pelos comentários e sugestões. Os erros remanescentes são de responsabilidades dos autores. 


\section{Apêndice A}

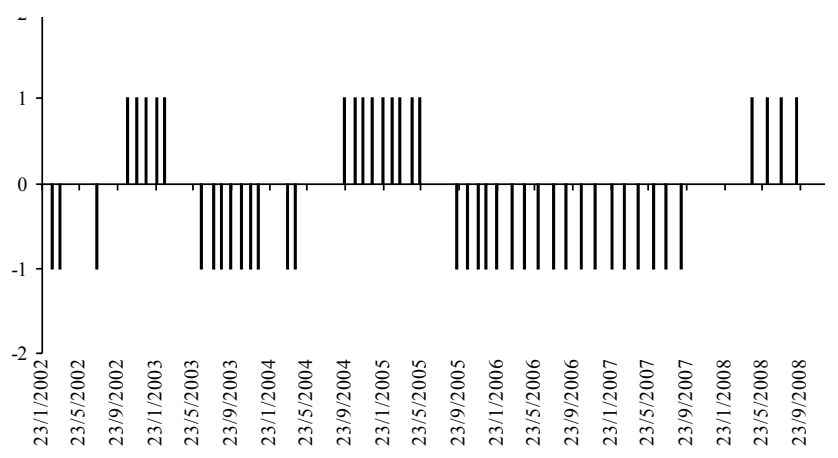

Figura A.1: Mudanças na taxa Selic-meta

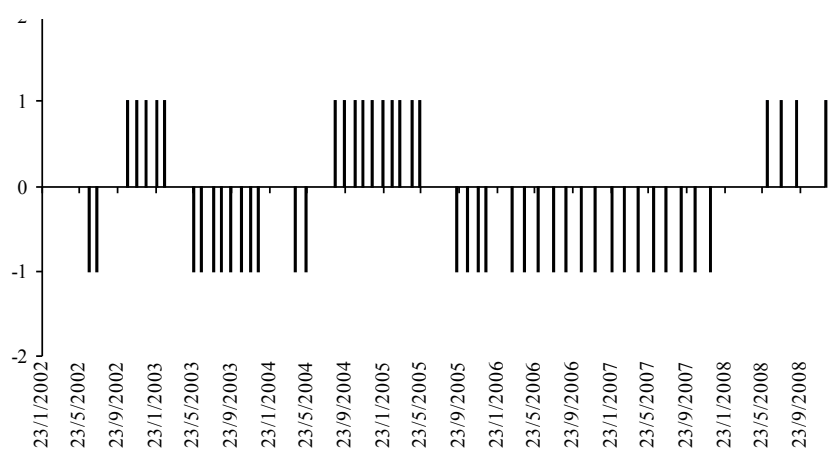

Figura A.2: Mudanças na taxa Selic-meta prevista pelo modelo 2

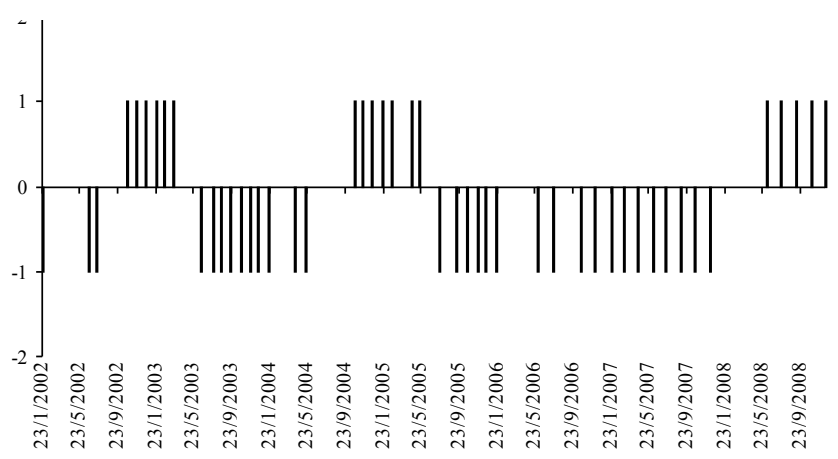

Figura A.3: Mudanças na taxa Selic-meta prevista pelo modelo 1 


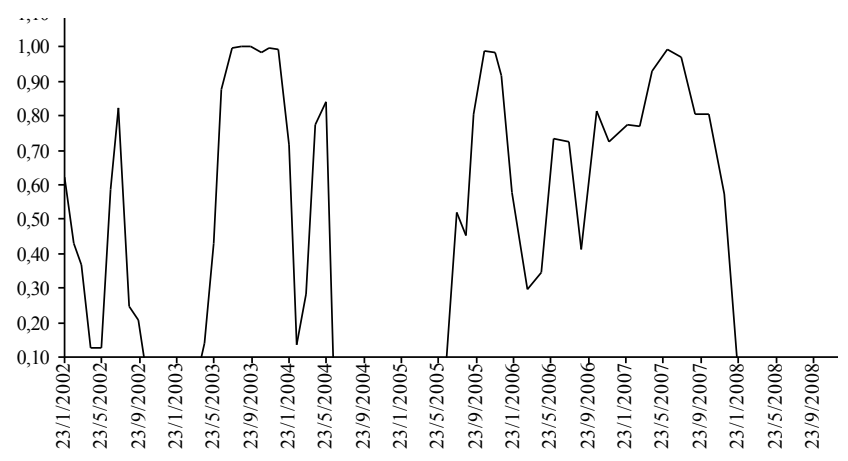

Figura A.4: Prob. de corte na taxa Selic-meta - modelo 1

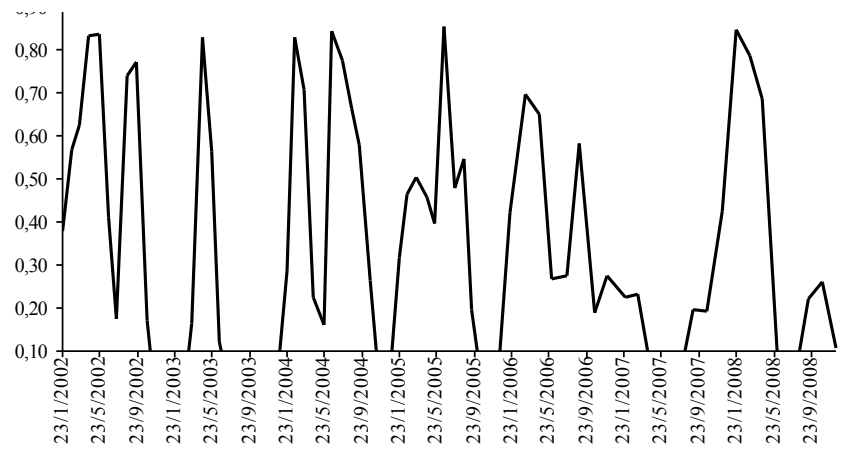

Figura A.5: Prob. de manter a taxa Selic-meta - modelo 1

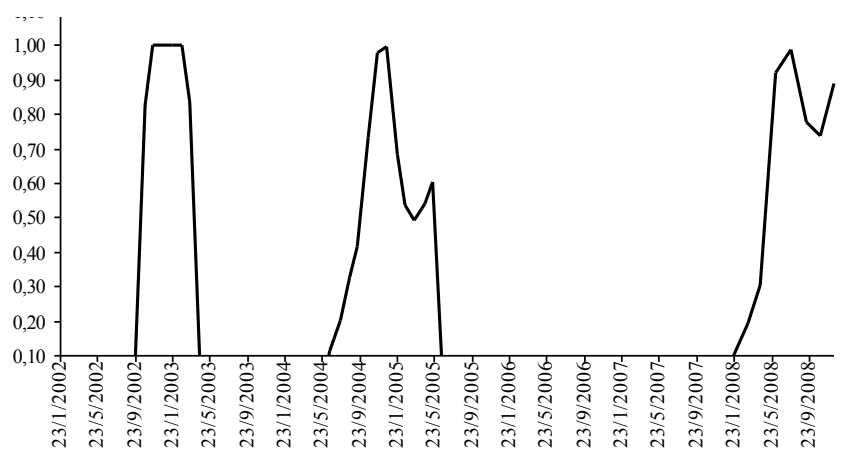

Figura A.6: Prob. de aumento na taxa Selic-meta - modelo 1 


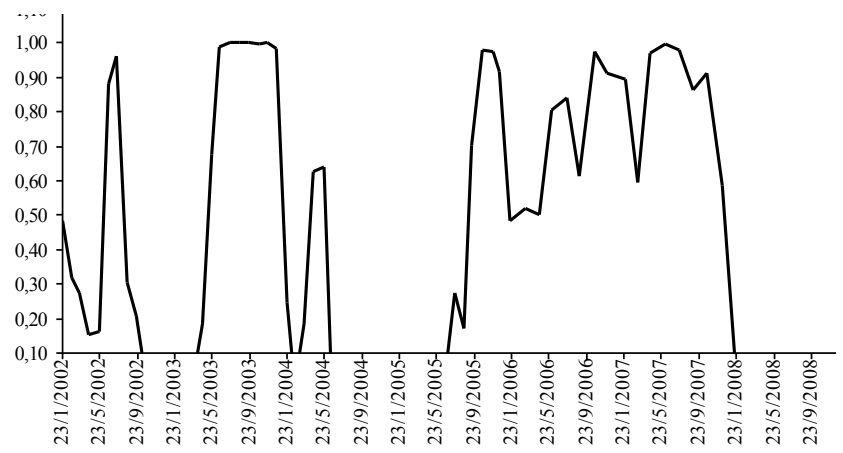

Figura A.7: Prob. de corte na taxa Selic-meta - modelo 2

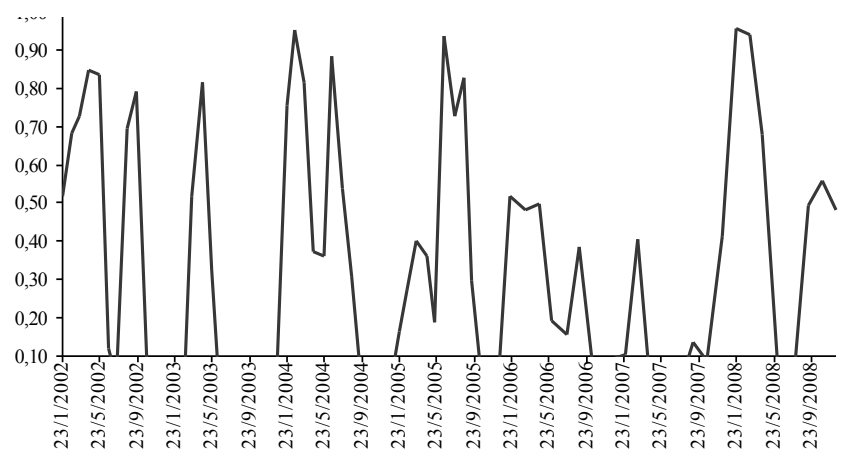

Figura A.8: Prob. de manter a taxa Selic-meta - modelo 2

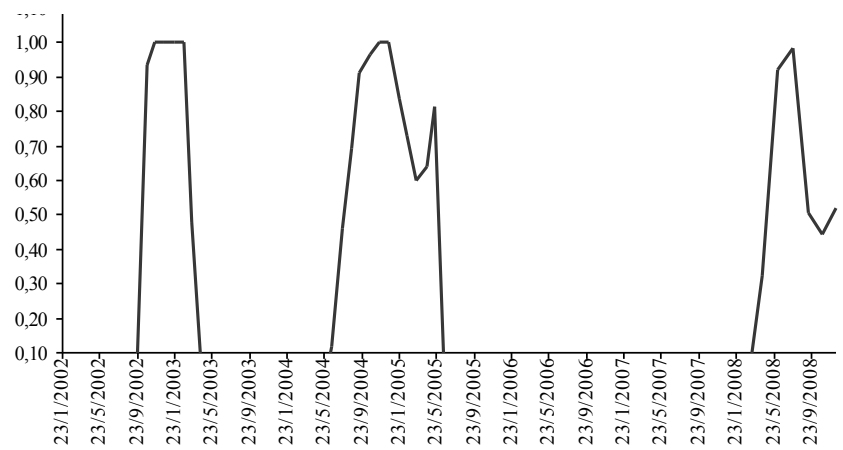

Figura A.9: Prob. de aumento na taxa Selic-meta - modelo 2 


\section{Referências Bibliográficas}

Barro, R. J. (2006), 'Milton friedman: perspectives, particularly on monetary policy', http://www.cato.org/events/monconf2006/barro.pdf. Acesso em: janeiro de 2007.

Cameron, A. C. \& Trivedi, P. K. (2005), Microeconometrics: methods and applications, Cambrige University Press.

Carneiro, D. D. \& Wu, T. (2004), 'Contas externas e política monetária', Revista Brasileira Economia 58(3), 301-323.

Clarida, R., Galí, J. \& Gertler, M. (2000), 'Monetary policy rules and macroeconomic stability: Evidence and some theory', The Quartely Journal of Economics 115(1), 147-180.

Cysne, R. P. (2005), 'Conservadorismo excessivo', Jornal do Brasil. Disponível em: http://www2.fgv.br/professor/rubens/homepage/artigos_e_reportagens_de_pol_econ/publicados/por_assunto. Acesso em: outubro de 2006.

Favero, C. A. \& Giavazzi, F. (2002), Why are Brazil's interest rates so high?, Working Paper 224, Università Bocconi, Innocenzo Gasparini Institute for Economic Research.

Figueiredo, F. M. R. \& Ferreira, T. P. (2002), Os preços administrados e a inflação no brasil, Trabalhos para Discussão 59, Banco Central do Brasil.

Hu, L. \& Phillips, P. C. B. (2004a), 'Dynamics of the federal funds target rate: a nonstationary discrete choice approach', Journal of Applied Econometrics 19(7), 851-867.

Hu, L. \& Phillips, P. C. B. (2004b), 'Nonstationary discrete choice', Journal of Econometrics 120(1), 103-138.

Maddala, G. S. \& Kim, I. M. (1999), Unit roots, cointegration, and structural change, Cambridge University Press.

McCallum, B. T. (1988), 'Robustness properties of a rule for monetary policy', Carnegie Rochester Conference on Public Policy 29.

McCallum, B. T. (2000), Alternative monetary policy rules: A comparison with historical settings for the United States, the United Kingdom and Japan, NBER Working Papers 7725, National Bureau of Economic Research.

Mendonça, H. F. (2007), 'Metas de inflação e taxa de juros no brasil: uma análise do efeito dos preços livres e administrados', Revista de Economia Política 27, 431-451.

Minella, A., Freitas, P. S., Goldfajn, I. \& Muinhos, M. (2002), Inflation targeting in Brazil: lessons and challenges., Technical report, Banco Central do Brasil.

Modenesi, A. (2008), Efeito convenção e inércia na taxa selic: uma estimativa da função de reação do bcb (2007-2007)., in 'I Encontro Internacional da Associação Keynesiana Brasileira'. 
Park, J. Y. \& Phillips, P. C. B. (2000), 'Nonstationary binary choice', Econometrica 68(5), 1249-1280.

Phillips, P. C., Jin, S. \& Hu, L. (2007), 'Nonstationary discrete choice: A corrigendum and addendum', Journal of Econometrics 141(2), 1115-1130.

Salgado, M. J. S., Garcia, M. G. P. \& Medeiros, M. C. (2005), 'Monetary policy during brazil's real plan: Estimating the central bank's reaction function', Revista Brasileira de Economia 59(1), 61-80.

Taylor, J. (1993), 'Discretion versus policy rules in practice', CarnegieRochester Conference Series on Public Policy 39, 195-214.

Taylor, J. B. (1979), 'Estimation and control of a macroeconomic model with rational expectations', Econometrica 47(5), 1267-86.

Taylor, J. B. (2000), 'Uso de reglas de política monetaria en economías de mercado emergentes', Seminario: Estabilización y Política Monetaria: La Experiencia Internacional. Banco de México. Disponível em: http://www.banxico.org.mx/tipo/publicaciones/seminarios/John\%20Taylor.pdf. Acesso em: setembro de 2006.

Teles, V. K. \& Brundo, M. (2006), 'Medidas de política monetária e a função de reação do banco central no brasil', XXXIV Encontro Nacional de Economia. 